\title{
SURGICAL TREATIMENT OF AN ASYMPTOMATIC LARGE CORONARY SAPHENOUS VEIN GRAFT ANEURYSM
}

Daniel Navia, MD, Guillermo Vaccarino, MD, Mariano Vrancic, MD, Fernando Piccinini, MD, and Eduardo Iparraguirre, MD, Buenos Aires, Argentina

Although the incidence of a minor degree of aneurysmal dilatation of a saphenous vein graft (SVG) 6 to 12 years after coronary artery bypass grafting has been found to be as high as $14 \%$, significant postoperative aneurysmal dilatation of an SVG is an unusual event. We report the surgical management of an unusual case of a large SVG aneurysm that was first seen as an asymptomatic mediastinal mass on a routine chest $\mathrm{x}$-ray examination.

Clinical summary. A 67-year-old white man with hypertension and previous myocardial infarction who had undergone double coronary artery bypass grafting 20 years previously was found to have an asymptomatic mass over the left border of the cardiac silhouette on a chest radiograph that was obtained as a part of a routine evaluation. During coronary revascularization in 1979 the patient had undergone placement of a reverse SVG to the left anterior descending coronary artery and a reverse SVG to the circumflex coronary artery. At that time no mention was made of the quality of the veins used for the grafts.

During the 20 years after the coronary artery bypass grafting the patient had no further cardiac symptoms. He stated that he was able to walk 3 to 4 miles and to play tennis with-

From the Cardiac Surgery Department, Instituto Cardiovascular de Buenos Aires, Argentina.

Received for publication July 6, 1999; accepted for publication Aug 6, 1999.

Address for reprints: Daniel Navia, MD, Cardiac Surgery Department, Instituto Cardiovascular de Buenos Aires, Blanco Encalada 1543/47, Ciudad de Buenos Aires (1428), Argentina (Email: don@lvd.com.ar).

J Thorac Cardiovasc Surg 1999;118:1122-3

Copyright (C) 1999 by Mosby, Inc.

$0022-5223 / 99 \$ 8.00+0 \quad \mathbf{1 2 / 5 4 / 1 0 2 0 8 9}$

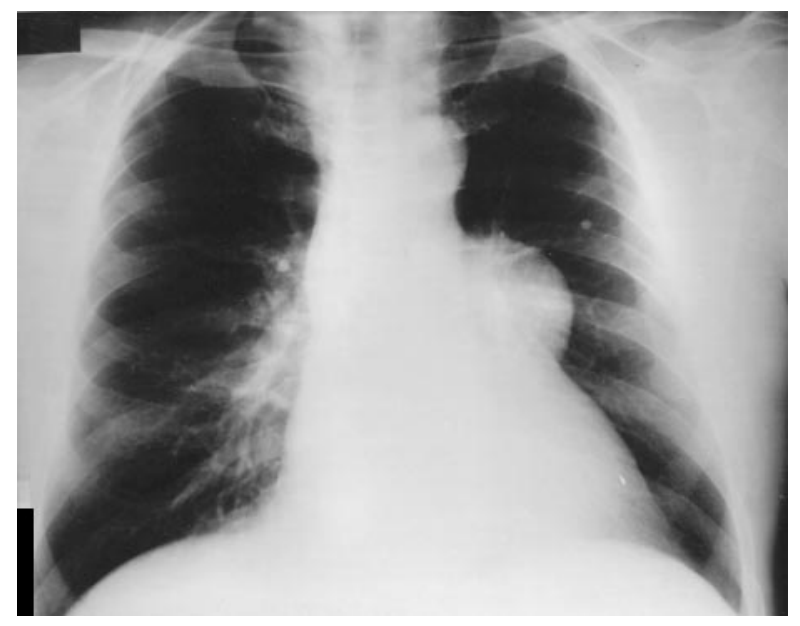

Fig 1. Chest radiograph demonstrating large mass adjacent to left heart border.

out difficulty. On physical examination the patient was a healthy appearing white man in no distress. Blood pressures were $130 / 70 \mathrm{~mm} \mathrm{Hg}$ in the right arm and $125 / 70 \mathrm{~mm} \mathrm{Hg}$ in the left. Heart rate was 60 beats/min. Pertinent findings included a grade 2 systolic ejection murmur at the apical region consistent with a flow murmur. No diastolic murmur was heard. The results of the examination and the laboratory data were within normal limits. The patient received medication with propranolol $40 \mathrm{mg} / \mathrm{d}$ and aspirin $100 \mathrm{mg} / \mathrm{d}$.

The chest radiograph demonstrated a large lateral mass adjacent to the left heart border (Fig 1). Further diagnostic evaluation included a computed tomographic scan of the chest, which revealed a large left lateral mediastinal mass 
abutting the lateral aspect of the left ventricle and into the left pleural space. After intravenous contrast material injection the tubular mass was enhanced to a similar degree as the aorta and pulmonary artery, confirming its vascular nature. A transesophageal echocardiogram showed a large aneurysmal formation at the lateral side of the heart and into the left pleural space, with Doppler flow signals and extensive intramural thrombotic material inside the formation. Catheterization of the right side of the heart, coronary angiography, and left and right ventriculography were performed. Pulmonary and wedge pressures were mildly to moderately elevated. Angiography revealed a patent SVG to the left anterior descending coronary artery with severe stenosis and moderate dilatations. The SVG to the circumflex coronary artery was poorly opacified with contrast material but appeared to be aneurysmally dilatated with no distal runoff. The right coronary artery was occluded with good distal vessels. Magnetic resonance imaging with gadolinium clearly defined the lesion as a vascular structure and yielded the appropriate dimensions in coronal and sagittal sections.

Because of severe coronary disease and the likelihood of complications, such as thrombosis, embolization, and fistula formation, vein graft aneurysm resection was performed. At the operation a large $(8 \times 8 \times 6 \mathrm{~cm})$ true aneurysm of the $S V G$ to the circumflex coronary artery was removed.

Surgical technique. The entire aneurysm was identified after opening the left pleural space after cardioplegic arrest was obtained. The aneurysm was opened through its long axis. It contained friable gelatinous tan-gray layered fibrin and thrombus, which appeared to have accumulated over a period of years. Retrograde coronary sinus cardioplegia helped to distinguish the distal anastomosis from inside the aneurysm; at the same time it was useful in flashing back all the fibrin and atherosclerotic material from the distal circumflex coronary artery to avoid perioperative myocardial infarction as a result of distal embolization. Bypasses were performed of the radial artery to the circumflex coronary artery, the left internal thoracic artery to the left anterior descending coronary artery, and a reverse SVG to the distal right coronary artery. The patient tolerated the operation well and was discharged after 6 days.
Discussion. True SVG aneurysms may be incidentally detected as an anterior mediastinal mass on chest radiography, but they are more frequently found as a result of clinical event, unstable angina or myocardial infarction ${ }^{1,2}$ caused by complete thrombosis or coronary artery emboli. ${ }^{3}$ The diagnosis of SVG aneurysm can be made by simple chest radiography in patients with previous coronary artery bypass grafting, but a diagnosis on this basis alone would be unlikely. Computed tomography, magnetic resonance imaging, and echocardiography have successfully assisted in the distinction of the true SVG aneurysm from pseudoaneurysms. Coronary angiography must still be pursued before any operation to determine vein graft patency and native coronary anatomy, however, and this procedure is also useful to confirm the diagnosis. ${ }^{4}$

Weakness of the graft wall near the valve sites has also been implicated as a cause. Here the muscle layer of the vein changes from circular to longitudinal, which may predispose toward aneurysmal dilatation.

Because the risk of rupture of the vein and the risk of embolization with the presence of cardiac symptoms appear to be high, we join others in recommending surgical excision of a large patent SVG aneurysm. ${ }^{5}$ Retrograde cardioplegia is useful not only to completely preserve the myocardium during the procedure but also to flash back all the intracoronary material to avoid distal myocardial embolization.

\section{REFERENCES}

1. Dzavik V, Lemay M, Chan KI. Echocardiographic diagnosis of an aortocoronary venous bypass graft aneurysm. Am Heart J 1989;118:619-21.

2. Sherry CS, Harms SE. MR imaging of pseudoaneurysm in aortocoronary bypass graft. J Comput Assist Tomogr 1989;13:426-9.

3. Taliercio CP, Smith HC, Pluth JR, Gibbons RJ. Coronary venous bypass graft aneurysm with symptomatic coronary emboli. J Am Coll Cardiol 1986;7:435-7.

4. Karwande SV, Sharp SD. Saphenous vein graft pseudoaneurysm presenting as a mediastinal mass. Tex Heart Inst J 1990;17:129-32.

5. Benchimol A, Harris CL, Desser KB, Fleming H. Aneurysms of an aorto-coronary artery saphenous vein bypass graft: a case report. Vasc Surg 1975;9:261-4. 